\title{
The 5th Cell-Based Assay and Bioanalytical Method Development Conference
}

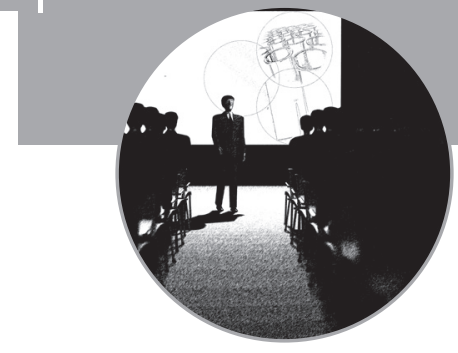

\section{5th Cell-Based Assay and Bioanalytical Method Development Conference}

Marriot Hotel, San Francisco, CA, USA 4-6 October 2010

Approximately 80 participants met at the Marriot Hotel, San Francisco, CA, USA, between the 4th and 6th October 2010 to share novel techniques and discuss the emerging approaches in the evolving field of cell-based assay and bioanalytical method development. This report highlights the discussion and summary of the meeting.

Between the 4th and 6th October 2010, US and European scientists across biopharmaceutical industry, government and CROs met in San Francisco to share emerging techniques and to discuss novel approaches in cell-based assay and bioanalytical method development.

The conference began with a joint plenary session to provide the common concepts and general updates, and was then followed by breakout sessions of the two groups to specifically focus on cell-based assay or bioanalytical method.

Among all the different types of cell-based and bioanalytical assays, three types of assay were mainly discussed at this conference:

- Potency assay;

- Immunogenicity assay;

- Pharmacokinetic (PK)/pharmacodynamic (PD) assays.

During the conference, the seminars and presentations were predominantly focused on four areas:

- Novel technologies;

- Bioanalytical troubleshooting-related issues;

- Current industrial trends and experiencesharing;

- New compliance requirements and regulatory strategies.

\section{Novel technologies}

- Design of experiments

During the workshop session, Jun Kim from Medimune, MD, USA, provided an introduction to design of experiments (DOE). He briefly examined the different kinds of designs available and reviewed cases demonstrating the utility and limitations of DOE in a bioanalytical method development. The highlights of his introduction included:

- The history of DOE;

- The comparison of the advantages and disadvantages of DOE versus ontario flow assessment techniques;

- The characterization of the types of designs;

- The review examples of the applications of DOE in bioanalytical development;

- An explanation of how to use screening design;

- The discussion on the response surface design;

- The development of fit-for-purpose and robust bioassay using DOE.

\section{- AlphaScreen ${ }^{\mathrm{TM}}$ technology}

Adriana Bajardi-Tacciol from Biogen Idec, MA, USA, illustrated a new application of AlphaScreen technology though the development and qualification of neonatal $\mathrm{Fc}_{\mathrm{C}}$-receptor $\left(\mathrm{Fc}_{\mathrm{C}}\right)$ binding assay. During her presentation, technical approaches in the method development and method validation were discussed. The data obtained from the comparison of assay formats indicated that the AlphaScreen technology can be employed to produce a sensitive and robust FcRn binding assay. In addition, the challenges associated with the nature of $\mathrm{F}_{\mathrm{C}-\mathrm{F}} \mathrm{Rn}$ interaction were also addressed in her presentation.

- Non-cell-based antibody-dependent cell-mediated cytotoxicity methods

In contrast to the traditional cell-based antibodydependent cell-mediated cytotoxicity (ADCC) assays, which are considered inherently complex,

\section{Mark Ma}

One Amgen Center Drive, MS B30E-3-C, Thousand Oaks, CA 91320 , USA

Tel.: +l 8054471000

E-mail:mhma@amgen.com
FUTURE
SCIENCE 
and are commonly associated with poor precision and reproducibility, Aaron Miller from Genentech, CA, USA, showed a novel ADCC assay development, which was a non-cell-based assay. It was developed to simultaneously measure binding of epitope to antibody and antibody to FcyRIIIA. This method combines the ease of use of an ELISA and the mechanism of action reflective nature of a cell-based ADCC assay, suggesting that it could serve as a surrogate for cell-based ADCC assays.

\section{- High-throughput platform technology} Michael Tovey from the Laboratory of Viral Oncology, Institute Andre Lwoff, Villejuif France, demonstrated a novel cell-based assay for quantification of the activity and antibody response to biopharmaceuticals. He presented the method development for the determination of both drug activity and the post-drug antibody response to a biopharmaceuticals. The method was developed by simply adding reporter engineered cells to the sample. The engineered cell expressed both firefly luciferase, controlled by a drug-responsive promoter, and the drug, Renilla luciferase, transcribed from a common inducible promoter. This unique high-throughput platform technology is rapid and precise; results were independent of cell number or viability and could be used to determine both postcomplementarity determining regions and post-Fc responses to a monoclonal antibodies $(\mathrm{mAb})$ in the same sample.

\section{Bioanalytical troubleshooting- related issues \\ - Effects of high mannose glycans}

The $N$-glycans present in the Fc region of $\mathrm{IgG}$ molecules have been shown to play a vital role in the biological functions of antibodies. Shen Chuang (Genentech) illustrated the evaluation of the effects of high mannose glycans (Man8/9 and Man5) on effector functions by presenting a study where in vitro assays, including $\mathrm{F} c \gamma$ receptor $(\mathrm{F} c \gamma \mathrm{R})$ binding, Clq binding, ADCC and complementdependent cytotoxicity assays were performed. Results of their study indicated that the antibodies with high mannose glycans had enhanced potency in FcyRIIIA binding and ADCC activity, but reduced potency in FcyRIIA/IIB binding and complement-dependent cytotoxicity activity.

\section{- Sensitive biomarker assays for dose-estimation studies}

Priti Hegde from Genentech presented a number of case studies, under the title: 'Advances in biomarkers for oncology: how to develop sensitive assays for dose estimation studies and improve safety of human studies'. He discussed the challenges of identifying a therapeutic dose of a large-molecule drug that was effective but not toxic. One way to improve confidence in dosing was development of PD biomarkers to understand the dosing curve. Utilizing the PD biomarkers enables researchers to examine the smallest amount of a therapeutic that is biologically active in patients. In Hegde's presentation he also shared the lessons he learnt establishing sensitive, robust and reproducible assays that could detect low pictogram levels of drugs in circulation and how to crossvalidate the assays in a biomarker lab. The presentation highlights were:

- The use of biomarkers, including why and when to use them;

- The lessons learnt in developing highly sensitive assays for biomarkers in oncology studies;

- The PD biomarkers to indicate that the drug activity is hitting target;

- The use of cell-based assays and ligand-binding assays to understand gene signaling, especially in animal models and translational medicine.

\section{- Quantification of protein therapeutics using generic immunoassays}

Robust analytical methods that accurately quantify protein therapeutics in biological fluids are required for PK and safety studies. However, different assay designs can yield different results, which may influence biotherapeutic development significantly. During the early phase of the protein therapeutic development, Yanmei Lu's group from Genentech intentionally measured drug concentrations using both generic and specific immunoassays. The generic assays were based on the detection of the Fc region of the therapeutic, and the specific assays used the target protein as the capture reagent. Two case studies were presented in which the specific assays quantified lower levels of therapeutic than the generic assays. In the first case, the quantification of a ligand-Fc fusion protein was substantially lower in the specific assay than in the generic assay. The cause of this discrepancy was found to be linked to aggregation of the ligand-Fc in vivo. In the second case, the lower concentration of human mAb detected by the specific assay was due to the presence of a naturally occurring soluble receptor as well as possible shedding of the receptor extracellular domain as a result of $\mathrm{mAb}$ treatment. Therefore, the specific assay detected 'free' $\mathrm{mAb}$ while the generic assay measured 'total' mAb. 


\section{Current industrial trends \& experience sharing}

Recently, the bioanalytical industry has significantly evolved through innovations and crossfunction collaborations. In this conference, some of the industrial trends and new approaches were introduced to the audiences.

\section{- New approach to measuring biological activity in drug exposure}

Shalini Gupta (Clinical Immunology, AMGEN, CA, USA), introduced a white paper regarding a novel approach to measuring bioactive biotherapeutics using cell-based assay. Using antibody interferon to detect biologically active levels of therapeutic drug (as opposed to neutralizing antibody detection) is an alternative approach to demonstrating whether antibodies are adversely affecting drug exposure. This could be a helpful method when high serum drug levels are present, thus making it difficult to detect a neutralizing antibody. This new approach provides a platform for determining if a drug is still biologically active when challenged by drug interference in neutralizing antibody assays.

Statistical approaches for appropriate cut point determination

Martin Kane from Human Genomes Sciences, MD, USA, discussed the challenges of applying the appropriate statistical analysis and cut-point determination in cell-based and bioanalytical assay development. The need for setting disease versus normal cut points and identifying the appropriate statistical analysis was also addressed. Kane presented how the US Pharmacopeia Chapter 111 parallelism analysis criteria were implemented, and the new strategies for dealing with the data that came from cell-based assays used in preclinical and clinical studies. His presentation specifically demonstrated the current practices of:

- Identifying the proper statistical analysis of cost-benefit analysis data for parallelism and relative potency calculations;

- Determining assay versus analyst variation and how to address it;

- Developing suitable acceptance criteria for cell-based assays to neutralizing antibodies;

- Statistical approaches to determine appropriate cut point, screening and specificity (confirmatory assay);

- Analyzing methods of normal versus non-normal data distribution;
- Implementing appropriate outlier removal methods, including new software and strategies that do not require a statistician.

\section{- Best practices for reliable assay transfer} to a CRO

As the volume of assays that are transferred to a contract laboratory or CRO increases, the problems are increasingly shown in assay variability post-transfer. The mixing of internal and external resources causes project and operational challenges, in addition to the technical hurdles. The presenters from the CRO and pharmaceutical companies shared the thoughts on how the assays should be transferred. Their presentation included:

- Industry experts discussing the pitfalls of outsourcing assays;

- A list of the questions you should consider asking from the sponsor company and outsourcing company to ensure the transfer goes smoothly;

- The variability of cell-based assays when transferring to a CRO laboratory;

- Information on bioassay technology transfer strategies to make the transfer smoother.

\section{- Risk-based approach to assessment of Fc effector function evaluation}

In release testing and potency assessment, it is imperative to understand the Fc function activity of the drug, specifically, critical quality attributes. The recent published white paper clearly lays out how to determine the critical quality attributes for $\mathrm{mAb}$ drugs and guidelines for categorizing and approaching those assessments. An Song from Genentech reviewed comments from the US FDA and EMA egulatory agencies regarding this approach, and addressed the strategies for how to perform Fc functional evaluations. Song also introduced one of the approaches for the assessment of Fc functionality - using cell-based assay and ligand-binding assays for regulatory approval.

\section{New compliance requirements \\ \& regulatory strategies}

\section{- The FDA perspectives on}

characterization \& chemistry, manufacturing

\& control challenges with

antibody-drug conjugates

Antibody-based conjugates are designed to deliver a therapeutic agent (e.g., cytotoxic drug) through a targeting moiety. Ideally, an 
antibody-drug conjugate remains nontoxic during circulation in vivo until it reaches its specific target site, where the drug is only activated once it is internalized to the target cell. Although such antibody-drug conjugates are simple in principle, significant chemistry, manufacturing and control (CMC)-related challenges have presented themselves during regulatory review and approval of these complicated products. The presentation from the FDA demonstrated the role of bioassays in the characterization of this class of products and some critical CMC issues.

\section{- Incurred sample reanalysis:}

FDA \& EMA regulatory expectations in bioanalytical methods

The FDA and EMA have each drafted guidelines for incurred sample reanalysis (ISR) to determine if the study results are reproducible over time. These guidelines provided clear guidances for both small- and large-molecule PK assays. Suzanne Brignoli from Genentech provided the introduction to ISR and highlighted the regulatory guidelines and industry best practices:

- Use ISR-standard operating procedures for quality assurance and as a reproducibility check for PK assays;

- Implement industry lessons learned for how to handle ISR failures;

- Understand how industry experts are addressing the cause of the failures;

- Implement strategies to overcome the common pitfalls.
The final joint session on the last day of the conference included an introduction to the Implementation of USP Bioassay Guidance for Parallelism Testing (Jun Kim, MedImmune, MA, USA) and a discussion of the controversies in antibody therapy for cancer ('Effector cells in ADCC and the Role of Complement', Josee Golay, laboratory of cellular and gene therapy $\mathrm{G}$ Lanzani, Ospedali Riuniti di Bergamo, Italy). The conference ended after the interactive sessions, including the ask-the-experts' session.

The general impression of the conference is reflected in the following thoughts: all good scientific endeavors happen with critical thinking, scientific discussion and information sharing. In the evolving fields of method development, scientists have a wealth of knowledge to share and a variety of topics to discuss. Over the past 5 years, this conference has been the only place to find the scientists who are discovering new approaches and solutions to the challenges in cell-based or bioanalytical assay development, and it will continue to be the main platform for cell-based assay and bioanalytical method development in the future.

\section{Financial \& competing interests disclosure} The author has no relevant affiliations or financial involvement with any organization or entity with a financial interest in or financial conflict with the subject matter or materials discussed in the manuscript. This includes employment, consultancies, honoraria, stock ownership or options, expert testimony, grants or patents received or pending, or royalties.

No writing assistance was utilized in the production of this manuscript. 